\title{
Biomarkers predict conversion from clinically isolated syndrome to multiple sclerosis
}

B iofluid and neuroimaging markers could help predict which patients with clinically isolated syndrome

(CIS) are at greatest risk for multiple sclerosis (MS), according to two recently published, large multicentre studies. About $85 \%$ of patients with MS first present with CIS, and $60-70 \%$ of individuals who experience an episode of CIS will eventually be diagnosed with clinically definite MS (CDMS) - yet the factors contributing to CIS-CDMS conversion are not completely understood.

"The identification of the factors influencing the conversion from CIS to CDMS is relevant for prognostication and early intervention strategies, and to improve our understanding of the pathological processes driving MS," explains Jens Kuhle. "Together with Giulio Disanto and other investigators, we aimed to build a large cohort of patients for whom long-term clinical follow-up data would be available."

In a study comprising 1,047 individuals with CIS from 33 centres across Europe, the researchers analysed serum and cerebrospinal fluid (CSF) samples in conjunction with demographics, clinical

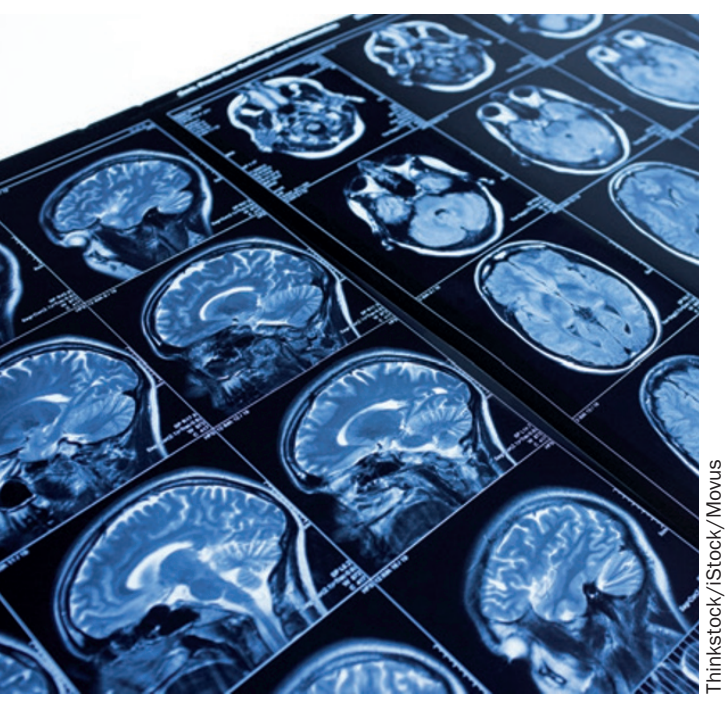

examination and neuroimaging to uncover factors that could predict CDMS.

The study participants were followed up for a median period of 4.31 years after CIS diagnosis. During this period, $60 \%$ of the patients converted to CDMS. The best predictors of conversion were a high number of hyperintense brain lesions on T2 MRI, the presence of CSF oligoclonal bands (OCBs), and a young age at CIS onset. For example, patients with CIS who were OCB-positive and had $>9$ T2-hyperintense lesions were at an $85 \%$ risk of experiencing a second demyelinating attack within 5 years, whereas only $30 \%$ of OCB-negative patients who had $\leq 2 \mathrm{~T}$-hyperintense lesions went on to develop CDMS.

The patients with CIS who had the lowest serum vitamin D levels also seemed to be at an increased risk of CDMS, but accounting for other variables attenuated this effect. "Our results could only partially confirm the vitamin D hypothesis and we believe this needs to be further explored," Kuhle comments. "Vitamin D is a potentially modifiable risk factor for MS and, if confirmed, the association could carry important therapeutic implications."

In contrast with a few previous studies, the findings by Kuhle and colleagues did not confirm tobacco use (as measured by serum cotinine levels) or antibody levels against the Epstein-Barr virus (EBV) as contributors to progression to CDMS. According to the authors, EBV antibodies might be a risk factor for CIS and MS rather than a progression marker.

Kuhle says that even though the team investigated a large number of clinical variables and molecular markers, there are still several other factors that could influence the likelihood of development of CDMS, possibly in interaction with genetic susceptibility. One such factor is the chitinase-3-like 1 (CHI3L1) protein, which has been previously implicated in CIS-CDMS progression. Manuel
Comabella and colleagues analyzed CHI3L1 level in CSF samples from 813 patients with CIS across 15 European MS centres. MS-associated disability was evaluated with the Expanded Disability Status Scale (EDSS).

The researchers found CHI3L1 predicted conversion independently of other strong covariates, such as brain MRI abormalities and CSF OCBs. "Interestingly, in our study, the CSF CHI3L1 level was the only significant independent risk factor for the development of neurological disability, with a hazard ratio close to four," says Comabella. In other words, CSF levels of CHI3L1 are a clear prognostic factor in patients with CIS.

"The association between CHI3L1 CSF levels and development of neurological disability, evaluated as time to reach EDSS score 3.0, is a novel finding," Comabella adds. Patients with high CHI3L1 levels typically had a particularly aggressive disease, with quick progression to CDMS and rapid development of disability.

The findings validate $\mathrm{CHI} 3 \mathrm{~L} 1$ as a prognostic marker for CIS-CDMS conversion. The team plans to extend the study by measuring CSF CHI3L1 levels in a prospective cohort of patients with CIS. "We also plan to investigate the added prognostic value of other CSF biomarkers in patients with CIS by measuring the levels of these markers in combination with CHI3L1," Comabella says.

Together, these findings sharpen the understanding of factors contributing to CIS-CDMS conversion, and might help delay or prevent disease progression.

\section{Hemi Malkki}

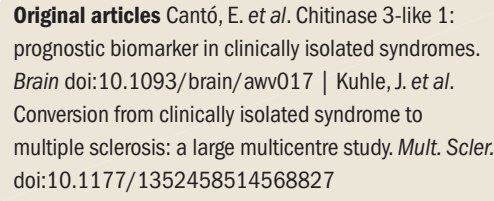
prognostic biomarker in clinically isolated syndromes. Brain doi:10.1093/brain/awv017 | Kuhle, J. et al. Conversion from clinically isolated syndrome to multiple sclerosis: a large multicentre study. Mult. Scler. doi:10.1177/1352458514568827 